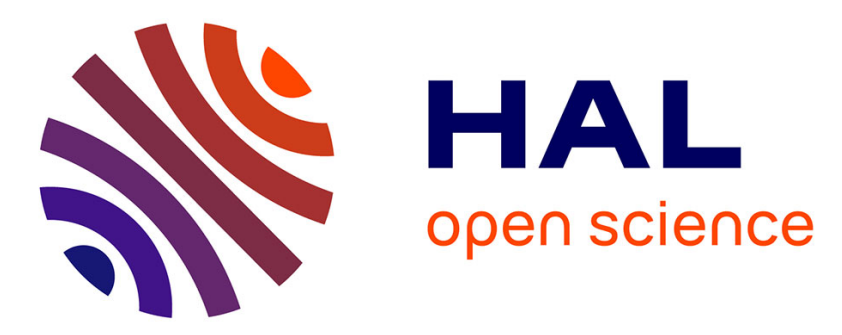

\title{
Foraging at the Edge of Chaos: Internal Clock versus External Forcing
}

S. C. Nicolis, J. Fernández, C. Pérez-Penichet, C. Noda, F. Tejera, O. Ramos, David J. T. Sumpter, E. Altshuler

\section{> To cite this version:}

S. C. Nicolis, J. Fernández, C. Pérez-Penichet, C. Noda, F. Tejera, et al.. Foraging at the Edge of Chaos: Internal Clock versus External Forcing. Physical Review Letters, 2013, 110 (26), pp.268104. 10.1103/PhysRevLett.110.268104 . hal-03013981

\section{HAL Id: hal-03013981 https://hal.science/hal-03013981}

Submitted on 11 Feb 2021

HAL is a multi-disciplinary open access archive for the deposit and dissemination of scientific research documents, whether they are published or not. The documents may come from teaching and research institutions in France or abroad, or from public or private research centers.
L'archive ouverte pluridisciplinaire HAL, est destinée au dépôt et à la diffusion de documents scientifiques de niveau recherche, publiés ou non, émanant des établissements d'enseignement et de recherche français ou étrangers, des laboratoires publics ou privés. 


\title{
Foraging at the Edge of Chaos: Internal Clock versus External Forcing
}

\author{
S. C. Nicolis, ${ }^{1 * *}$ J. Fernández, ${ }^{2}$ C. Pérez-Penichet, ${ }^{2}$ C. Noda, ${ }^{2}$ F. Tejera, ${ }^{2}$ O. Ramos, ${ }^{3}$ D. J. T. Sumpter, ${ }^{1}$ and E. Altshuler ${ }^{2}$ \\ ${ }^{1}$ Mathematics Department, Uppsala University, Uppsala 751 06, Sweden \\ ${ }^{2}$ Physics Faculty, "Henri Poincaré" Group of Complex Systems, University of Havana, Havana 10400, Cuba \\ ${ }^{3}$ Institut Lumière Matière, UMR5306 Université Lyon 1-CNRS, Université de Lyon 69622 Villeurbanne, France
} (Received 28 February 2013; published 27 June 2013)

\begin{abstract}
Activity rhythms in animal groups arise both from external changes in the environment, as well as from internal group dynamics. These cycles are reminiscent of physical and chemical systems with quasiperiodic and even chaotic behavior resulting from "autocatalytic" mechanisms. We use nonlinear differential equations to model how the coupling between the self-excitatory interactions of individuals and external forcing can produce four different types of activity rhythms: quasiperiodic, chaotic, phase locked, and displaying over or under shooting. At the transition between quasiperiodic and chaotic regimes, activity cycles are asymmetrical, with rapid activity increases and slower decreases and a phase shift between external forcing and activity. We find similar activity patterns in ant colonies in response to varying temperature during the day. Thus foraging ants operate in a region of quasiperiodicity close to a cascade of transitions leading to chaos. The model suggests that a wide range of temporal structures and irregularities seen in the activity of animal and human groups might be accounted for by the coupling between collectively generated internal clocks and external forcings.
\end{abstract}

DOI: 10.1103/PhysRevLett.110.268104

PACS numbers: 87.23. $-\mathrm{n}, 05.45 .-\mathrm{a}, 05.65 .+\mathrm{b}$

Activity rhythms occur both within individual animals and in social groups, with examples ranging from humans through insects down to micro-organisms [1-4]. This emergence of collective activity is in many respects reminiscent of chemical and physical processes involving many degrees of freedom $[5,6]$. In group-living animals, selfsustained activity oscillations can be a consequence of either exogenous or endogenous dynamics, or a combination of these. One important exogenous factor is the daynight cycle and associated changes in temperature $[7,8]$. Animals living in groups also have endogenous activity cycles [9]. For example, in ants isolated individuals exhibit an apparently random pattern in their activity, while colonies show periodic activity bouts [10]. These observations suggest that ant activity is autocatalytic in the sense that the activation of an individual increases with the number of already active ants, giving rise to a positive feedback [2].

When considering the interaction between exogenous and endogenous factors in social activity, a major challenge is in quantifying dynamics in natural conditions. Ant foraging, one of the paradigmatic subjects in the field of social animals, has been examined quantitatively in controlled laboratory experiments $[11,12]$ and for short temporal windows in the field $[13,14]$. Here we are able to quantify the activity of foraging ants in a natural environment over several days with a resolution of a few seconds using ad hoc infrared sensors placed at the entrance to the nests [15].

We first develop a mathematical model of activity cycles for social groups, incorporating both endogenous interactions and exogenous forcing. We consider a situation where there is a flow $\phi$ of individuals, e.g., ants leaving the nest.
These individuals enter an active state, denoted $A$, with a probability $f(A, I)$ that increases with the number of active individuals and decreases with the number of retired, inactive ones, the latter denoted $I$. The transition from active to inactive occurs at a constant rate. In the absence of external forcing the time evolution of active and inactive individuals is then

$$
\frac{d A}{d t}=\phi f(A, I)-k A \quad \frac{d I}{d t}=k A-k^{\prime} I,
$$

where $k$ is the transition rate of individuals from the active to the inactive state, and $k^{\prime}$ is the rate at which inactive individuals cease participating in the process due to the presence of constraints such as crowding effects. The function $f(A, I)$ that determines the probability of becoming active is modeled as a Hill-like function

$$
f(A, I)=\frac{K^{2}+A^{2}}{2 K^{2}+A^{2}+I^{2}},
$$

where $K$ is a threshold. When $I$ is small almost all individuals become active, but as $I$ increases the retired ants serve to inhibit further recruitment. This type of model is generic and applicable to a wide range of situations beyond ant's foraging, from social (e.g., gaze following in humans [16]) to intracellular (e.g., calcium oscillations [1]) dynamics.

The steady state solutions of Eqs. (1) and (2) and their stability can be solved analytically (see 1 in Supplemental Material [17]). The analysis reveals that the system undergoes one Hopf bifurcation at a particular value of $k$ [Fig. 1(a)], where the variables start to oscillate at a frequency equal to 

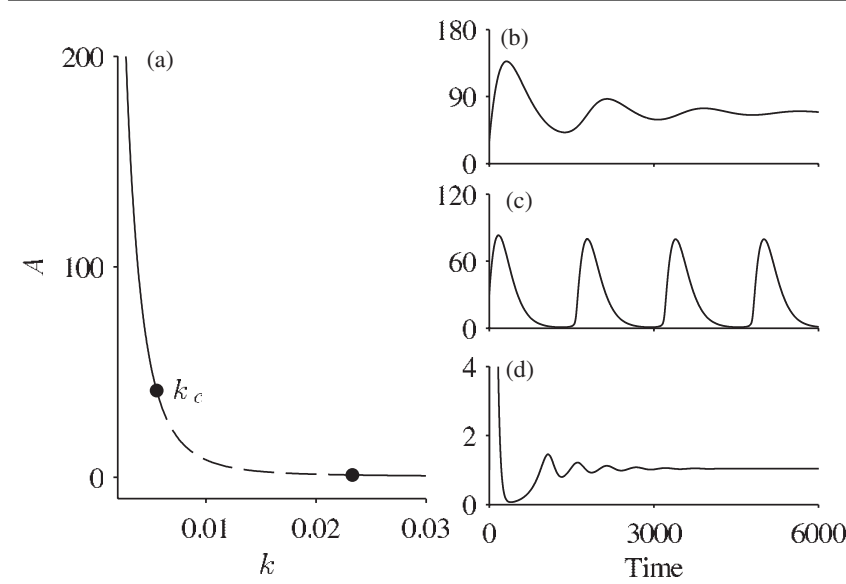

FIG. 1. Bifurcation diagram of the steady states of $A$ against the parameter $k$ (a). Time behavior of active individuals before the first bifurcation point $k_{c}(k=0.0045)(\mathrm{b})$, after the first bifurcation point $(k=0.007)(\mathrm{c})$, and after the second bifurcation point $(k=0.025)(\mathrm{d})$. Other parameter values are $\phi=1$, $k^{\prime}=0.003$, and $K=1$.

$\Omega=\sqrt{k k^{\prime}+\left[2 \phi K^{2} A_{s}\left(\left(k^{2} / k^{\prime}\right)-k^{\prime}\right) /\left(2 K^{2}+A_{s}^{2}+I_{s}^{2}\right)^{2}\right]}$. Figures 1(b) and 1(c) show how the number of active individuals changes through time before and after the bifurcation. A closer study reveals that for $k$ values substantially larger than $k_{c}$, a reverse Hopf bifurcation takes place, beyond which oscillatory behavior disappears and the steady state is again stabilized [Fig. 1(d)]. In what follows we will focus on $k$ values above and relatively close to the first bifurcation point $k_{c}$. Around this point there is temporal asymmetry in group activity, with a rapid increase in activity at the start of a cycle, followed by a slower return to inactivity [Fig. 1(c)].

To incorporate exogenous forcing we add a time dependent component to the flow term. Equations (1) become

$$
\frac{d A}{d t}=\phi[1+T(t)] f(A, I)-k A, \quad \frac{d I}{d t}=k A-k^{\prime} I,
$$

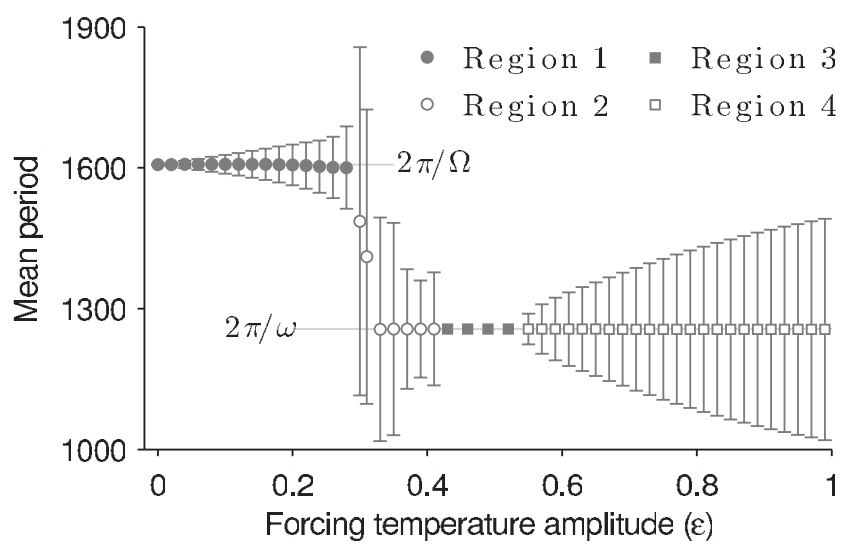

FIG. 2. Mean period of oscillations of $A$ as a function of the amplitude of the forcing $T(t)$. The vertical bars correspond to the standard deviation. Other parameter values are $k=0.007, k^{\prime}=$ $0.003, K=1, \phi=1$, and $\omega=0.005$. where $T(t)$ accounts for changes in the environment over time. In much of what follows, we use a simple sinusoidal function of amplitude $\epsilon$ and frequency $\omega, T(t)=\epsilon \sin \omega t$. Equations (3) define a periodically driven nonlinear oscillator [18]. The coupling between endogenous interactions and exogenous forcing produces a rich variety of dynamics. Figure 2 provides examples of different activity cycles as $\epsilon$ is increased, keeping the frequency $\omega$ constant. The dots and squares are located at the numerically observed mean periods, whereas the vertical bars represent the standard deviations around the means. In the upper branch, the mean frequency is equal to the intrinsic oscillation frequency, $\Omega$, whereas in the lower branch it is equal to the forcing frequency, $\omega$. Four different regions can be identified:Region 1: Provided $\epsilon<0.3$ the mean frequency is equal to $\Omega$. However, as soon as $\epsilon$ is not strictly zero the oscillations exhibit increasing deviations from this intrinsic period. Region 2: For $\epsilon \sim 0.3$ the deviations become especially pronounced. The mean period changes then suddenly to that of the forcing, but first the oscillations do not follow this periodicity exactly. Region 3: At $\epsilon_{1} \sim 0.43$ till about $\epsilon_{2} \sim 0.52$ full entrainment at the forcing period is observed. Region 4: Finally, beyond $\epsilon_{2}$, the oscillations cease again following the forcing period.

Figure 3 depicts the time dependence of $A$, the phase space plot, and the Poincaré surface of section (recorded at time intervals separated by the mean period) for regions 1 to $4[19,20]$. We conclude that in region 1 the behavior is quasiperiodic, [Fig. 3(a)], while in region 2 it is chaotic [Fig. 3(b)]. In these two regions the group's intrinsic activity cycle enhances its flexibility in responding to external forcing. The group adapts quickly to changes in the dynamical environment, with a rapid coordinated

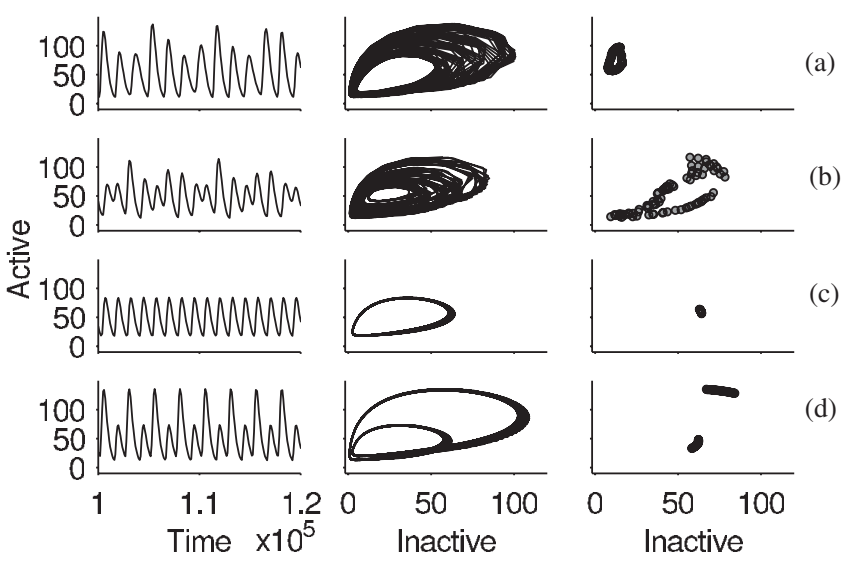

FIG. 3. Activity versus time, phase space plots and Poincaré surface section recorded at time intervals separated by the mean period for four different $\epsilon$. (a) A quasiperiodic behavior $(\epsilon=$ 0.26 ), (b) a chaotic behavior ( $\epsilon=0.36$ ), (c) a periodic behavior with period equal to the forcing $(\epsilon=0.46)$, and (d) a periodic behavior with a period twice the forcing $(\epsilon=0.76)$. Other parameter values as in Fig. 1(c) and $\omega=0.005$. 
increase in activity followed by a slower decrease. In region 3 the oscillations are strictly periodic [Fig. 3(c)] and the group activity cycles follow the external forcing. Region 4 is also periodic but with a period twice that of the forcing period [Fig. 3(d)]. Here, the group first over- then under-responds to the exogenous forcing.

Given the above analysis we predict that social groups that have evolved to deal with changes in the external environment should exhibit dynamics consistent with regions 1 or 2, where they respond flexibly. We also predict that these groups will exhibit rapid activity increases and slower decreases. We now test these predictions in ants.

We monitored the activity profile of the ant species Atta Insularis, a leaf-cutter ant endemic of Cuba [21]. A sensor, consisting of a ring with a reflective inner wall, was calibrated in order to establish the correspondence between the number of interruption signals per unit time and the number of ants crossing the ring either way per unit time (i.e., the flow of ants getting in or out from the nest). These measurements were defined as the ants' activity. Near the activity sensor we positioned a digital thermometer (also linked to our electronics) able to collect the environmental temperature data with a resolution of 0.5 Celsius. One of the time series is depicted in Fig. 4. Each day, the temperature near the nest's entrance is seen to increase relatively fast, while the cooling down occurs more slowly. The activity record shows that each day, the minimum of activity corresponds approximately to the maximum temperature and vice versa; i.e., the ants avoid foraging in the hottest hours.

Our measurements support the model prediction of an asymmetry in the initiation and cessation of activity. The foraging activity increases each day quickly when it becomes cool, but decreases more slowly as the temperature increases again. The asymmetry between temperature and activity can be quantified in the following way (see also 2 in the Supplemental Material [17]): if, for a 24 hour



FIG. 4 (color online). Temperature and activity time series on one nest for approximately 8 full days. Upper panel: Temperature time series. Lower panel: Activity time series (activity is measured as number of measured pulses every 36 seconds). period between two minima of activity we calculate the difference between the position of the maximum minus half the length of the time interval (i.e., 12 hours), the maximum is always shifted to the left. If we average these shifts for several days and express the result as a percent of the duration of one day, we get $-16 \pm 6 \%$ for the data depicted in Fig. 4. The asymmetry in the daily activity cannot be trivially linked to the asymmetry in the time evolution of temperature: if one assumes, for example, that the number of foraging ants is proportional to $1-T_{n}(t)$ (where $T_{n}(t)$ is the normalized environmental temperature) within a standard pheromone deposition-evaporation computational model [22], the experimental asymmetry in the activity record is not reproduced.

Figure 4 shows temperature is not purely sinusoidal. For a more realistic comparison with the data we therefore fit the experimental temperature with a sum of sinusoidal terms which for our purposes can be truncated to $T(t)=$ $\sum_{i=1}^{8} \epsilon_{i} \sin \left(\omega_{i} t+\varphi_{i}\right)$, where $\epsilon_{i}, \omega_{i}$, and $\varphi_{i}$ are the different amplitudes, frequencies, and phases of the external signal. We also extend the model in order to include the stochasticity inherent in the phenomenon. Figure 5(a) represents the evolution in time of the fitted forcing and



FIG. 5. Monte Carlo simulation with a deterministic forcing fitted from the data with a sum of eight sinuses (a), autocorrelation of activity's experimental and simulated data (b), probability distribution of the frequency of activity signal of the experimental and simulated data (c), and probability distribution of the variable $A \times(T-\bar{T})$ (d). Parameter values as in Fig. 1(c) but for a forcing equal to $\sum_{i=1}^{8} \epsilon_{i} \sin \left(\omega_{i} t+\varphi_{i}\right)$ with $\epsilon_{i}, \omega_{i}$, and $\varphi_{i}$ fitted to the data in Fig. 4: $\epsilon_{1}=0.1359, \omega_{1}=0.004387$, $\varphi_{1}=2.197, \quad \epsilon_{2}=0.01797, \quad \omega_{2}=0.003825, \quad \varphi_{2}=-1.974$, $\epsilon_{3}=0.03699, \omega_{3}=0.0005156, \varphi_{3}=-1.599, \epsilon_{4}=0.03763$, $\omega_{4}=0.001148, \quad \varphi_{4}=-0.2025, \quad \epsilon_{5}=0.04034, \quad \omega_{5}=$ $0.008723, \quad \varphi_{5}=-2.639, \quad \epsilon_{6}=0.6858, \quad \omega_{6}=0.002471$, $\varphi_{6}=1.167, \quad \epsilon_{7}=0.6865, \quad \omega_{7}=0.00246, \quad \varphi_{7}=-1.925$, $\epsilon_{8}=0.01727, \omega_{8}=0.005573, \varphi_{8}=0.02816$. 
resulting activity in a Monte Carlo simulation run for the same time span as the experiment. This figure resembles closely the experimental figure (Fig. 4) and displays the same properties such as a near antiphase shift between the forcing and activity as well as irregular intensity peaks of activity. The model also predicts the asymmetry between the increase and the decrease of activity within each day giving a peak asymmetry of $-19 \pm 7 \%$, near the experimental value. As for the autocorrelation function, we can see in Fig. 5(b) that it becomes flat about the same time for the data and the simulation. The probability distributions of the frequencies [Fig. 5(c)] and of the variable $A(T-\bar{T})$ tend also to be very similar, giving an antiphase relation for negative values and an in-phase one for positive values [Fig. 5(d)]. It is interesting to notice that the mean amplitude $\left(1 / 8 \sqrt{\sum_{i=1}^{8} \epsilon_{i}^{2}}\right)$ of temperature variability is 0.123 , a value that corresponds in Fig. 3 to a point near the chaotic regime, which produces flexible responses to perturbations.

The experiments support the model predictions of a social "waking up" and "winding down" dynamics, in the form of rapid increases followed by slower decreases in activity. This asymmetric cycle is familiar to many human societies, with important meetings planned at the start of the working day and more informal low-level activities taking place in the evenings. While in humans this pattern might be attributed to forward planning, for the ants it is the result of local interactions [23]. This conclusion on complex decision patterns - and flexibility in particularemerging from the underlying evolution laws is in line with some ideas developed in the recent book by Viswanathan et al. [24].

Rather than becoming entrained to a regime and following passively the external signal without any discrimination of environmental perturbations, the group is instead capable of maintaining a degree of flexibility and produce its own characteristic response. As a result, activity cycles can occasionally jump out of synchronization, resulting in surprisingly high or low levels of activity. The parameters compatible with our experiment suggest that the system operates in a regime of quasiperiodicity close to a cascade of transitions leading to chaos $[1,25]$. Anecdotal evidence supports the existence of such fluctuations in human activities [26]. An interesting model prediction that we have not observed experimentally here, relates to region 4 in our model. In this region activity levels systematically under- and overshoot the stimulus, resulting in somewhat dysfunctional activity cycles. We expect that all four types of activity rhythm predicted by our model may be observable in humans and other animals.

E. A. thanks P. Altshuler for measuring foraging ants speeds in the wild, D. Maza for useful comments and S. Luke for advice in the modification of MASON. We acknowledge funding from ERC grant ID-CAB. *snicolis@math.uu.se

[1] A. Goldbeter, Biochemical Oscillations and Cellular Rhythms: The Molecular Bases of Periodic and Chaotic Behavior (Cambridge University Press, Cambridge, England, 1996).

[2] S. Goss and J. L. Deneubourg, Insect Soc. 35, 310 (1988).

[3] B. J. Cole, J. Insect. Behav. 4, 129 (1991).

[4] J. Aschoff, M. Fatranská, H. Giedke, P. Doerr, D. Stamm, and H. Wisser, Science 171, 213 (1971).

[5] A. Arenas, A. Díaz-Guilera, J. Kurths, Y. Moreno, and C. Zhou, Phys. Rep. 469, 93 (2008).

[6] V.S. Anishchenko, V. Astakhov, A. Neiman, T. Vadivasova, and L. Schimansky-Geier, Nonlinear Dynamics of Chaotic and Stochastic Systems: Tutorial and Modern Developments (Springer, New York, 2002).

[7] F. Ruano, A. Tinaut, and J. Soler, Behav. Ecol. 11, 396 (2000).

[8] K. Human, S. Weiss, A. Weiss, B. Sandler, and D. Gordon, Environ. Entomol. 27, 822 (1998).

[9] L. Conradt and T. Roper, Proc. R. Soc. Lond. B Biol. Sci. 267, 2213 (2000).

[10] B. J. Cole, Proc. R. Soc. Lond. B Biol. Sci. 244, 253 (1991).

[11] A. Dussutour, S.C. Nicolis, J.-L. Deneubourg, and V. Fourcassie, Behav. Ecol. Sociobiol. 61, 17 (2006).

[12] K. Johnson and L.F. Rossi, J. Theor. Biol. 241, 360 (2006).

[13] M. Burd and N. Aranwela, Insect Soc. 50, 3 (2003).

[14] A. John, A. Schadschneider, D. Chowdhury, and K. Nishinari, Phys. Rev. Lett. 102, 108001 (2009).

[15] C. Noda, J. Fernández, C. Pérez-Penichet, and E. Altshuler, Rev. Sci. Instrum. 77, 126102 (2006).

[16] A. C. Gallup, J. J. Hale, D. J. T. Sumpter, S. Garnier, A. Kacelnik, J. R. Krebs, and I. D. Couzin, Proc. Natl. Acad. Sci. U.S.A. 109, 7245 (2012).

[17] See Supplemental Material at http://link.aps.org/ supplemental/10.1103/PhysRevLett.110.268104 for model steady state and stability analyses and for data analysis.

[18] J. Guckenheimer and P. Holmes, Nonlinear Oscillations, Dynamical Systems, and Bifurcations of Vector Fields (Springer-Verlag, New York, 1983).

[19] M. G. Rosenblum, A.S. Pikovsky, and J. Kurths, Phys. Rev. Lett. 76, 1804 (1996).

[20] R. Jensen, Am. J. Phys. 70, 607 (2002).

[21] E. Altshuler, O. Ramos, Y. Núñez, J. Fernández, A. Batista-Leyva, and C. Noda, Am. Nat. 166, 643 (2005).

[22] S. Luke, C. Cioffi-Revilla, L. Panait, K. Sullivan, and G. Balan, Simul-trans Soc. M S 81, 517 (2005).

[23] S. Camazine, N. R. Franks, J. Sneyd, E. Bonabeau, J.-L. Deneubourg, and G. Theraulaz, Self-Organization in Biological Systems (Princeton University Press, Princeton, NJ, 2001).

[24] G. M. Viswanathan, M. G. da Luz, E. P. Raposo, and H. E. Stanley, The Physics of Foraging: An Introduction to Random Searches and Biological Encounters (Cambridge University Press, Cambridge, England, 2011).

[25] M. J. Feigenbaum, J. Stat. Phys. 19, 25 (1978).

[26] D. Helbing, Quantitative Sociodynamics: Stochastic Methods and Models of Social Interaction Processes (Springer, New York, 2010). 